\title{
Important progress and future prospects for studies on urban public recreational space in China
}

\author{
YU Ling ${ }^{1,2,3},{ }^{*}$ LIU Jiaming ${ }^{1,2}$, LI TaO $^{4,5}$
}

1. Key Laboratory of Regional Sustainable Development Modeling, Institute of Geographic Sciences and Natural Resources Research, CAS, Beijing 100101, China;

2. College of Resources and Environment, University of Chinese Academy of Sciences, Beijing 100049, China;

3. Center for Spatial Information Science, The University of Tokyo, Tokyo 113-8656, Japan;

4. School of Geographic Science, Nanjing Normal University, Nanjing 210023, China;

5. Jiangsu Center for Collaborative Innovation in Geographical Information Resource Development and Application, Nanjing 210023, China

\begin{abstract}
As the main sites of urban residents' recreational activity in a mass leisure era, urban public recreational space (UPRS) is the primary component of urban public spatial systems. To satisfy current demands for the construction of livable cities and to meet the challenges of UPRS development in an urbanizing context, a scientific and systematic review of the latest developments in UPRS research is important for developing this field. Based on 1264 papers from the China National Knowledge Internet and the Web of Science from 1985 to 2018, this study examined research developments on UPRS in China. Through a quantitative literature analysis, we divided UPRS-related research in China into three stages of development: the starting period (1985-2000), the growth period (2001-2009), and the consolidation period (2010-2018). Next, we identified nine "hot" research topics on UPRS and reviewed developments in each of these. These topics were the classification of UPRS, spatial structure and layout, comprehensive evaluation, planning and development, the impacts of UPRS development, spatial-temporal evolution laws and mechanisms, space imagery features, sustainable utilization, and governmental decision making on UPRS. We also systematically reviewed the research methods and spatial scales used in UPRS-related studies in China. In response to deficiencies in existing research, we put forward several suggestions regarding future research: Chinese UPRS studies should focus on basic theoretical research; construct a new research system that accounts for Chinese characteristics, based on interdisciplinary and multilevel spatial integration; develop a humanistic philosophy and build management systems for UPRS that can lead to the development of world-class cities based on a global vision.
\end{abstract}

Keywords: era of mass leisure; urban tourism; urban public recreational space; quantitative analysis; China

Received: 2018-07-12 Accepted: 2018-11-12

Foundation: National Natural Science Foundation of China, No.41071110

Author: Yu Ling (1992-), PhD Candidate, specialized in the research of urban tourism, tourism geography, planning and design of recreational space. E-mail: yul.15b@igsnrr.ac.cn

"Corresponding author: Liu Jiaming, Professor, E-mail: liujm@igsnrr.ac.cn 


\section{Introduction}

Internationally, the first studies on "recreational space" appeared in the 19th century. In 1979, the concept of "recreational space" was introduced to China by researchers in Taiwan, China (Huang and He, 1979). Because recreational activities have increasingly become the main content of people's leisure consumption, "recreational space" has been distinguished from spaces for other social and economic activities.

In terms of spatial hierarchy, the concept and connotations of urban public recreational space (UPRS) have not been uniformly defined, and scholars have used multiple interpretations. From a unidimensional spatial hierarchy perspective, UPRS is regarded as a material space defined as an open space, building, or facility that is located in a city or suburb, where visitors can enter and leave freely, that has some recreational function, such as entertainment, rest, shopping, sightseeing, communication, exercise, or tourism (Wu et al., 2003). From a dual-dimensional spatial hierarchy perspective, UPRS is regarded as a binary space system that combines a material recreational space and an urban socioeconomic recreational space (Feng, 2006b). Scholars, taking this latter perspective, analyze the spatial structures while also paying attention to recreational activities and behavior within the UPRS (Qin, 2003). From a three-dimensional spatial hierarchical perspective, UPRS is considered a complex spatial system composed of a material space formed by recreational venues and facilities, an action space occupied by recreationists, and a space of capital and power under the control of government departments and economic management organizations (Ye and Bao, 2009a).

The continual growth of rapid urbanization, UPRS is an important aspect of assessing urban social civilization and the quality of life of residents. Clearly, the planning and development of UPRS are significant for improving the livability of the city and increasing urban residents' happiness. The imbalance between the surging demand for participation in recreation and the inadequate amount of UPRS severely restricts urban residents' happiness and the livability of the city. To solve the problems (e.g. over-crowdedness, road congestion, and the misuse of UPRS) caused by high-intensity recreational activities in specific time periods (e.g. weekends and holidays), scholars have carried out many studies on UPRS from multiple perspectives. This previous work has made great progress in understanding UPRS in China and has put forward some constructive suggestions about the layout, rational planning, space design, and service fairness of UPRS.

The development and expansion of cities, the advancement of industry, and changes in people's recreational activities have had new impacts and brought new challenges for the planning and layout of UPRS, the supply of facilities, and the design of experiences and activities. In particular, in the era of mass leisure, the convenience, comfort, and high quality of residents' recreational experiences are essential for the construction of livable cities and the sustainable development of cities. Questions of how to adapt to the changes in the role of UPRS in urban development and how to coordinate the relationship between recreational space management and city construction have increasingly attracted the interest of scholars across various disciplines, such as urban planning, tourism geography, and landscape architecture. To cope with the growing problems of UPRS development in this new era, a scientific and systematic review of the latest developments in UPRS research is urgently needed to promote the development of this research field.

This article presents the results of a quantitative analysis of data from the China National 
Knowledge Internet and the Web of Science, drawing on 1264 articles on the theme of "recreational space in China" that were published from January 1985 to September 2018. This study examines and summarizes research developments on UPRS in China, including the main contents and topics covered, research methods, and spatial scales of relevant studies, to identify current "hot" research topics, existing problems, and future directions for UPRS research.

\section{Characteristics and contents of UPRS research in China}

This section reviews the main features and contents of UPRS research in China and summarizes the research methods and spatial scales used in this existing work.

\subsection{Main characteristics of UPRS research in China}

The main characteristics of UPRS research in China include phase characteristics and hierarchical structure characteristics.

\subsubsection{Phase characteristics of UPRS research in China}

From 1985 to 2018, the number of UPRS-related articles published in the journals increased (Figure 1). Especially after 2000, researchers paid more attention to UPRS in the context of the leisure tourism era. Based on the number and growth rate of articles published per year, we divided China's UPRS-related articles into the following three stages of development:

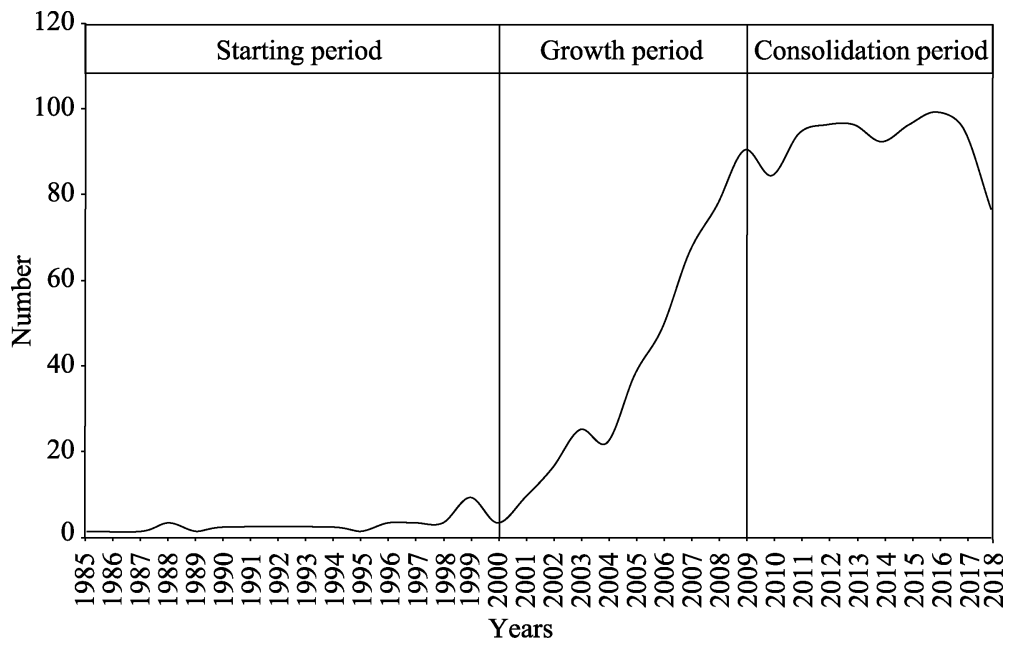

Figure 1 Stage characteristics of urban public recreational space research in China from 1985 to 2018

(1) Starting period (1985-2000). During this period, with the reform and opening up of policy, as well as domestic socioeconomic growth, China's leisure and recreation industry began to develop. At the same time, the concept of recreational space was introduced in research in China. Research contents and topics were mainly based on the analysis of the contents, methods, historical developments, and recreation planning theory found in Western research. Although the literature published during this period was relatively limited, these pioneering achievements served as good preparation for later research.

(2) Growth period (2001-2009). During this period, with China's tremendous socioeconomic development, there were changes in people's lifestyles and attitudes toward recreation. People's awareness increased regarding the importance of daily participation in recreational 
activities. Residents' demands for recreational fields, facilities, and environments increased. At the same time, studies related to UPRS received widespread attention from scholars. Work in this field grew along with the rapid growth of residents' demand for recreation. Relevant research results increased rapidly during this phase.

(3) Consolidation period (2010-2018). Along with the change in residents' views on recreational consumption, recreational activities grew from being an interest of a minority group to being a pursuit of the broader public, becoming a necessary part of residents' lives. Attention to urban recreation became a constant focus of governments, enterprises, and academics. Research on the ecological environment, recreational activities, space optimization of residents' habitual recreational spaces (e.g. waterfront spaces, urban greenways, and urban parks) became "hot" topics for academic research. Compared with the growth period, the depth and breadth of the research has improved continuously. Studies with different target groups appeared, including work on children's recreational space (Chen et al., 2010) and sports recreational space (He, 2014), for example.

\subsubsection{Hierarchical structure characteristics of UPRS research in China}

This section draws on a visual approach to identify research hotspots and predict development trends using the co-occurrence frequency of key words. Figure 2 presents the knowledge network of UPRS research in China, based on the co-occurrence of key words in the top 10 highly cited articles for each year from 1985 to 2018. In Figure 2, each key word node represents a hot topic. The radius of these nodes reflects the degree of concern about

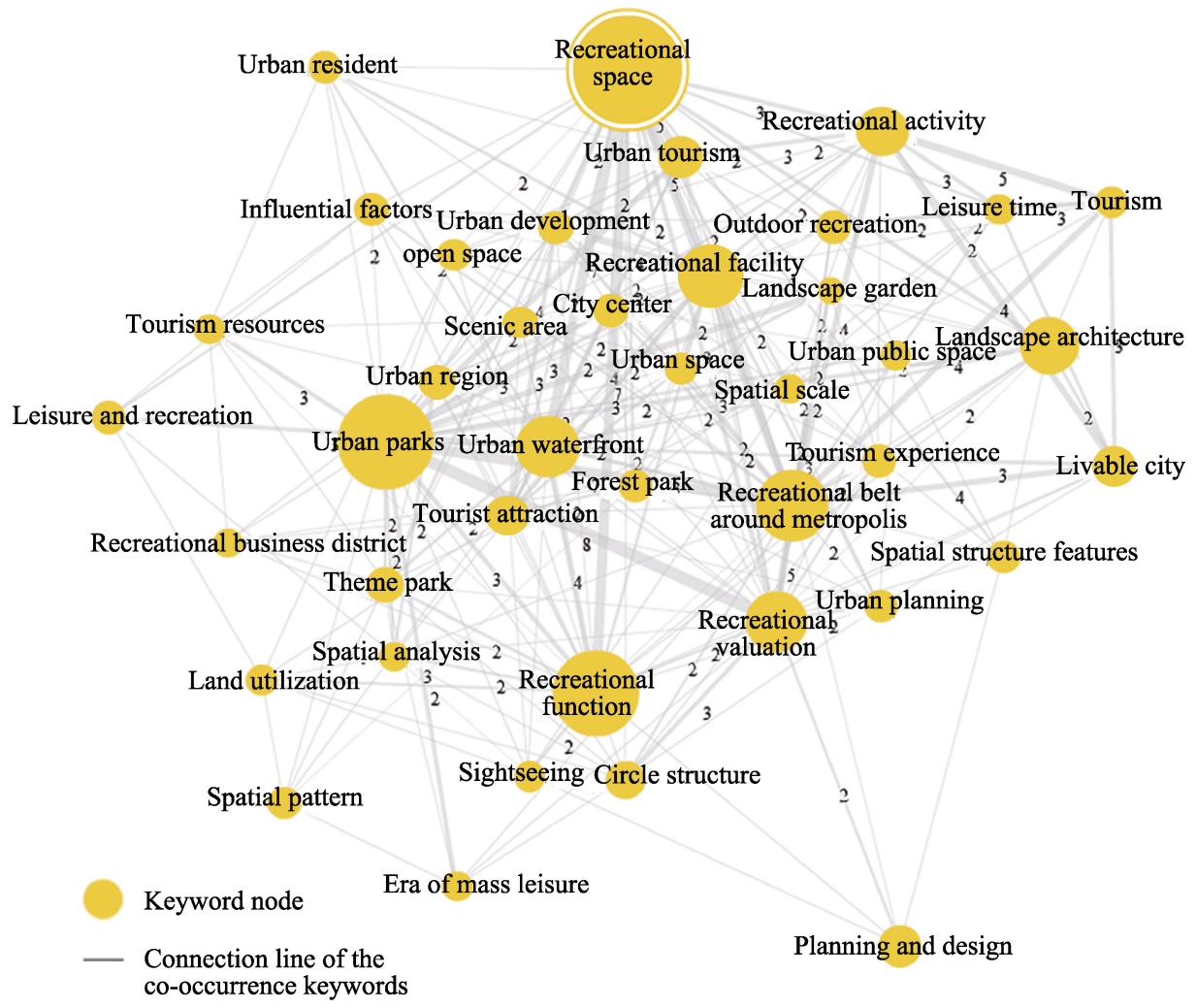

Figure 2 Knowledge network of urban public recreational space research in China based on the co-occurrence of key words (1985-2018) 
that topic; the larger the radius, the more attention that topic was given. The lines connecting the nodes indicate key word co-occurrence; the thicker the connection line, the higher the frequency of key word co-occurrence. The number on the connection line indicates the frequency of co-occurrence.

Figure 2 illustrates the framework of UPRS research in China, presenting a hierarchical structure with features similar to a grade structure known as "stars holding the moon." The core is "recreational space," which is analogous to the moon. Around "recreational space" are various research issues that can be regarded as the stars; these include urban parks, landscape architecture, recreational belt around a metropolis, tourism resources, urban tourism, and recreational functions.

\subsection{The topics and contents of UPRS research in China}

According to the knowledge network of UPRS research in China based on the co-occurrence of key words and the hierarchical structure of UPRS research in China, the present article summarizes the topics and trends of UPRS research (Table 1). The identified research topics included the classification of UPRS, spatial structure and layout, comprehensive evaluation, planning and development, impacts of UPRS development, spatio-temporal evolution laws and mechanisms, space imagery features, sustainable utilization, and governmental decision making.

Table 1 Direction of urban public recreational space research in China

\begin{tabular}{|c|c|c|}
\hline Research topic & Research tendency & Percentage $(\%)^{\mathrm{a}}$ \\
\hline $\begin{array}{l}\text { Classification of urban } \\
\text { public recreational space }\end{array}$ & $\begin{array}{l}\text { According to different classification standards, including the } \\
\text { service object's attributes, function of recreation, recreational } \\
\text { activity, spatial form, attributes of tourism resources, scope of } \\
\text { service, geographical location, and composite attributes }\end{array}$ & 4.19 \\
\hline $\begin{array}{l}\text { Spatial structure and lay- } \\
\text { out }\end{array}$ & $\begin{array}{l}\text { e.g. Elements, structural models, distribution laws and contrib- } \\
\text { uting factors, and ideal structural patterns }\end{array}$ & 12.58 \\
\hline Comprehensive evaluation & $\begin{array}{l}\text { Evaluation of recreational space quality, assessment of accessi- } \\
\text { bility, comprehensive evaluation of the quality of the recrea- } \\
\text { tional environment, assessment of the recreation value }\end{array}$ & 16.06 \\
\hline $\begin{array}{l}\text { Planning and development } \\
\text { strategies }\end{array}$ & $\begin{array}{l}\text { Optimal allocation, management of recreational land, factors } \\
\text { influencing allocation }\end{array}$ & 38.37 \\
\hline $\begin{array}{l}\text { Impacts of urban public } \\
\text { recreational space devel- } \\
\text { opment }\end{array}$ & $\begin{array}{l}\text { Relationship between UPRS and urban residents' happiness, the } \\
\text { impact of urban recreational business districts on urban devel- } \\
\text { opment }\end{array}$ & 6.17 \\
\hline $\begin{array}{l}\text { Spatio-temporal evolution } \\
\text { laws and mechanisms }\end{array}$ & Characteristics, laws, dynamic mechanisms of evolution & 3.09 \\
\hline Space imagery features & Space imagery features related to urban residents and visitors & 4.03 \\
\hline Sustainable utilization & $\begin{array}{l}\text { Reasonable utilization of urban industrial heritage sites, historic } \\
\text { streets, and industrial abandoned lands; the post-competition } \\
\text { utilization of Olympic venues; integration of UPRS compo- } \\
\text { nents }\end{array}$ & 5.46 \\
\hline $\begin{array}{l}\text { Government decision mak- } \\
\text { ing on UPRS }\end{array}$ & $\begin{array}{l}\text { Government administrative departments choosing different } \\
\text { integrative schemes based on realistic conditions in cities }\end{array}$ & 1.03 \\
\hline Other & $\begin{array}{l}\text { Economic issues, cultural connotations, recreation consumption } \\
\text { patterns, intergenerational transformation of recreationists' be- } \\
\text { havior (Dong et al., 2015); recreation conflict in UPRS (Wong } \\
\text { and Yu, 2012); economic valuation of the amenities of UPRS } \\
\text { (Jiao and Liu, 2010) }\end{array}$ & 9.02 \\
\hline
\end{tabular}

${ }^{a}$ Percentage of all articles from 1985 to 2018 on each specific research topic 


\subsubsection{Classification of UPRS}

Classification is the first fundamental step in research on UPRS, playing a supporting role in further theoretical, research and practical planning. Driven by different research objectives, scholars have adopted different classification systems to categorize UPRS. According to the service object's attributes, UPRS has been divided into two categories: for residents vs. for external tourists and residents (Wu et al., 2003). In terms of the recreational function, UPRS has been categorized as commercial recreational space, self-supporting recreational space, or public supply recreational space (Ma, 2005). According to the recreational activity, UPRS has been classified by park type, fitness track type, sports venue type, and other types (Wei et al., 2016). In terms of spatial form, physical space has been categorized as planar, block, or linear recreational space; for behavioral space, the form has been divided into planar space (e.g. park walks, public recreational squares, and public reading rooms), and three-dimensional space (e.g. climbing, skydiving, and diving) (Qin, 2003). According to the attributes of tourism resources, UPRS has been divided into recreational spaces for natural sightseeing, humanistic sightseeing, artificial entertainment, sports, and folk experience (Li et al., 2011). According to the scope of service, UPRS has been classified as regional, urban/town, community, or indoor recreational spaces (sorted from large to small types) (Qin, 2003). In terms of geographical location, UPRS has been divided into urban, suburban, and urban fringe recreational spaces.

Most of the above methods have classified UPRS on a single dimension. UPRS is an interdisciplinary research topic with multiple, varying dimensions, so it is difficult to reach a consensus on unified classification criteria. From the perspective of structuralism, Ye Shengtao and Bao Jigang (2009a) used duality to describe the elements of the urban recreational spatial structure, creating an index of urban recreational spatial morphology. These scholars established a classification framework known as the ROP-ENCS. This framework divided UPRS into four main types (experience-leading, nature-leading, culture-leading, and service-leading). In future, the classification of UPRS will necessarily be more complicated to account for continual change in residents' recreational preferences.

\subsubsection{Spatial structure and layout of UPRS}

Along with increases in people's recreational time, the type and amount of UPRS have also increased systematically. Studies on the spatial patterns and distribution regularity of UPRS provide a theoretical basis for reasonably allocating and efficiently managing UPRS. The study of the structure and layout of UPRS systems can be summarized using the following three components:

(1) Study of the elements of recreational space systems. From the perspective of spatial form, the UPRS system is composed of three parts - namely, point (sightseeing spots), line (recreational corridor), and surface (recreational center) (Feng, 2010). In terms of supply and demand, the UPRS system is composed of recreational supply elements (government, economic organization), recreational demand elements (urban recreationists), and the connection between the supply and demand elements (recreational channels and recreational routes) (Xiao and Song, 2008). Some scholars view this differently, arguing that the elements connecting supply and demand are recreational sites and facilities (Ye and Bao, 2009a).

(2) Study of the structural model of recreational space systems. The spatial pattern of UPRS varies from city to city, and some changes can be seen within the same area across 
different periods. Initially, there was little UPRS and it was all a single type. The center of UPRS was often found in a single place, revealing a single-core structural model (e.g. the West Lake as the former core recreational center of Hangzhou). Even now, some small cities need only one large urban park to meet the recreational demands of most people. However, with socioeconomic development and the rising need for recreation, urban recreational centers are often distributed across various places, and UPRS systems have multi-core or group structural models; this is often the case in metropolises such as Beijing, Shanghai, or Chongqing.

The urban recreation spatial structural model is also largely influenced by the urban spatial form and the natural environment. Where there are rivers or seashores, the UPRS structure tends to take the form of a band (e.g. the Seine River in Paris or both sides of the Yellow River). Certain kinds of topography and geomorphology also influence UPRS structures. For example, in metropolitan areas located on plains (e.g. Chengdu, Beijing), UPRS forms a circular structure pattern (Yu, 2003; Feng, 2010). However, because UPRS systems each have various types and functions, these systems' structure usually takes a composite form (Table 2). The structural models can provide planners and policy makers with a theoretical basis that can be used in UPRS planning and assessment. However, in actual application, there may be discrepancies, to a certain extent, from any UPRS structural model because of the models' limitations and many other factors such as the city size, natural environ-

Table 2 Composite structure models of urban public recreational space

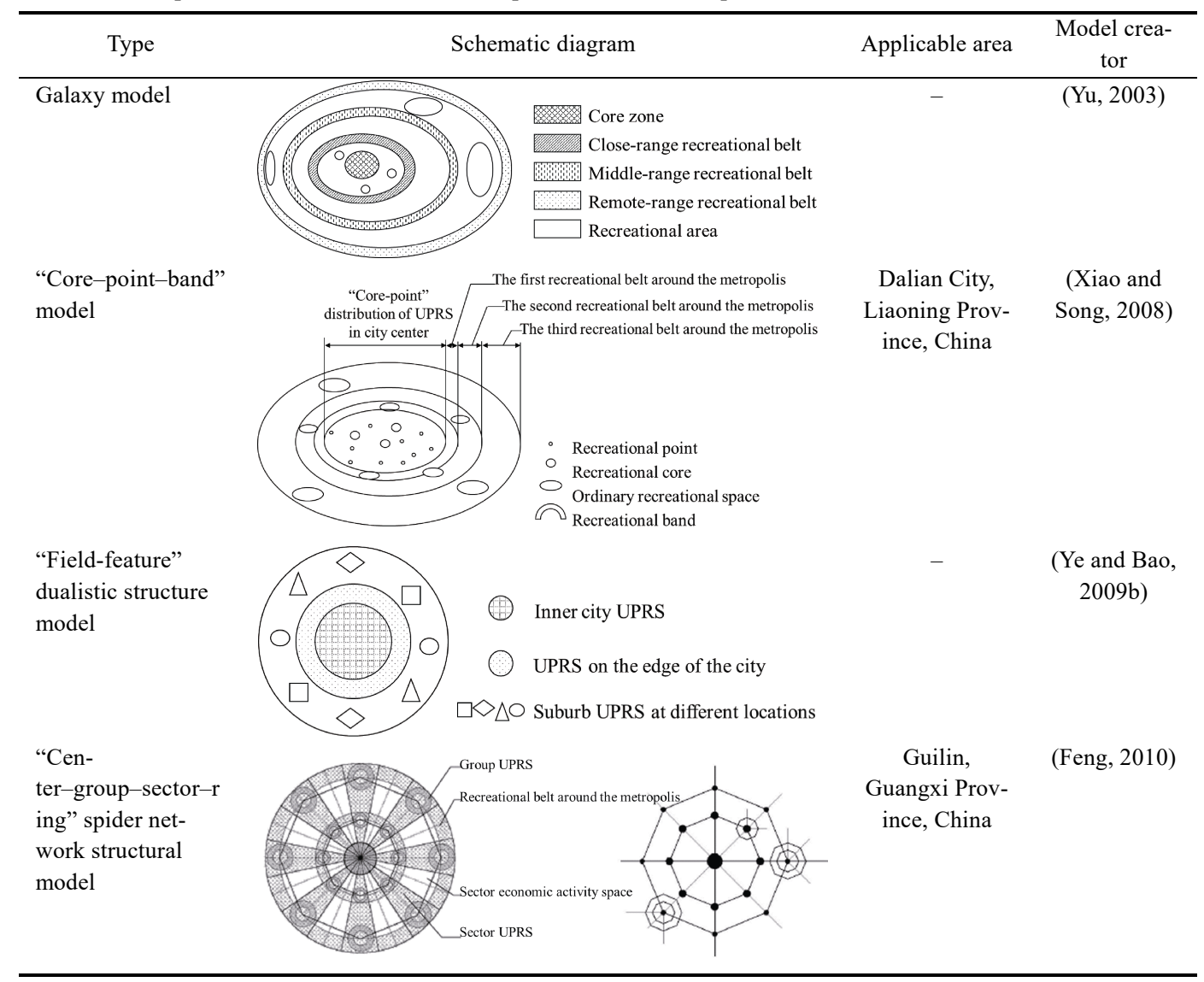


ment, and socioeconomic development.

(3) Study of spatial distribution laws and contributing factors of UPRS. Studies of spatial distribution patterns and the factors influencing UPRS can provide a reference for reasonable urban development planning. Most existing studies do some work to describe the distribution characteristics, spatial aggregation patterns, evolution rules, and driving mechanisms of UPRS for specific case studies, using geographical statistics, nearest-neighbor indicators, and spatial analysis with geographic information system (GIS) software. Such case studies have involved Beijing, Shanghai, Wuhan, Chongqing, Dalian, Nanchang, Shaoxing, and other cities (Wu, 2001; Gu and Bao, 2002; Wu and Jia, 2002; Xiao and Song, 2008; Huang et al., 2009; Huang et al., 2010; Liu et al., 2010b; Wu and Tian, 2012; Li, 2014). The results of these analyses have shown that the UPRS around the city generally appears in spatial clusters. However, there are significant differences between different types of aggregation. The average distance from UPRS to the urban center varies widely, and this is related to whether the UPRS is of the artificial entertainment type, humanistic sightseeing and sport type, or natural sightseeing type. The analysis of the driving mechanisms of spatial distribution features indicates that tourism resources, distance from the city center, landform, rivers, and other factors have an important impact on the distribution of UPRS (Su et al., 2004; Liu et al., 2010b). In recent years, to analyze UPRS in depth, some scholars have investigated the effect of a single influencing factor on the spatial pattern of UPRS (e.g. examining the impact of a low-carbon public transport system on UPRS spatial distribution) (Wang et al., 2017a).

\subsubsection{Comprehensive evaluation of UPRS}

The evaluation of UPRS plays an essential guiding role in UPRS planning and development. Studies on the evaluation of UPRS have mainly included the following four aspects:

(1) Evaluation of recreational space quality. Recreational space quality is a relatively new research topic in the field of UPRS analysis and evaluation. Recreational space quality depends on whether the space can effectively meet the needs of its users. Potential factors influencing recreational space quality involve various aspects, such as recreational facilities support, recreational activity planning, recreation image recognition, and recreation operation guarantee (Xin and $\mathrm{Hu}, 2016$ ). Existing studies have mainly used the recreational perceptions (Feng et al., 2009) and satisfaction index (Xiao and Du, 2011; Lian et al., 2012; Mao et al., 2013) of visitors to assess UPRS quality. An indicator assessment model has been established, considering the characteristics of the specific UPRS and the main service object (e.g. the elderly, children, or residents). The Analytic Hierarchy Process method was designed to quantitatively evaluate recreational space quality using data from questionnaires and interviews.

(2) Assessment of UPRS accessibility. Quantification of UPRS accessibility is a prerequisite for evaluating and reasonably allocating UPRS with consideration for equity in decision making and planning. Accessibility is a commonly used indicator for quantitatively measuring the degree of resistance people need to overcome to arrive at a particular destination. The main resistance factors include distance, cost, and time (Yu et al., 1999). To assess accessibility, scholars initially calculated a simple ratio between supply of and demand for UPRS within the research area. After GIS tools were widely implemented, the scope of ac- 
cessibility studies was greatly enriched. Most existing studies, based on data from various datasets, have measured accessibility using ArcGIS software. Some researchers have used traffic and distance data (Yue et al., 2016; Wang et al., 2017a). Others have argued that the functionality and attraction to recreationists of UPRS should also be taken into account in the evaluation (Ren and Wan, 2016), and other scholars have included road networks and population distribution data ( $\mathrm{Li}$ and Liu, 2009; Yang and Shi, 2016). Some researchers working in this area have employed the Two-Step Floating Catchment Area method to appraise UPRS accessibility (Gu et al., 2017; Wei, 2017).

(3) Evaluation of the recreational environment and service quality. Because the recreational environment and service quality of UPRS are key factors that significantly affect recreationists' satisfaction and consumption behavior, it is crucial to understand and evaluate both these factors. Through empirical and quantitative analysis, scholars have tried to analyze recreationists' perceptions and evaluations of the recreational environment and service quality. Depending on the particular context, researchers have selected different influencing factors and created specific assessment models. After assessing the recreational environment and service quality in Beijing, Shanghai, Tianjin, and 13 other metropolises in China, one study concluded that the three best cities in terms of recreational environment and service quality were Nanjing, Beijing, and Chengdu (Gan, 2004). Shanghai had low scores on the comprehensive evaluation of environmental quality and the functional level of ecological recreational space services. The main reason for these low scores was not that the amount of recreational space was insufficient, but rather the functional loss caused by low service coverage and uneven spatial distribution ( $\mathrm{Li}, 2014)$.

(4) Recreational valuation of UPRS. The study of the recreational valuation of UPRS has important implications for the allocation and management of UPRS. The travel cost method is a popular evaluation method for assessing the recreational value of UPRS (Li et al., 2010b). Some scholars have also conducted comparative analyses of the recreation value of urban ecological recreational space by dynamically observing recreationists' spontaneous recreation behaviors. The results of these studies have shown that areas with water activities year-round had the highest recreation value, followed by grasslands and woodlands, and that woodland recreational spaces had the highest recreational value for the elderly, whereas middle-aged people preferred water-area recreational spaces, and young people and children preferred grassland recreational spaces (Sun et al., 2016b).

\subsubsection{Planning and development of UPRS}

The planning and construction of UPRS are important parts of urban planning and development. It is necessary to carry out reasonable planning and design to ensure that the elements of recreational spaces are in harmony with urban functions. Planning and design in China has increasingly attracted the interest of scholars working in various disciplines, who have carried out systematic studies from different perspectives.

In tourism geography, scholars have usually paid attention to morphological structures, distribution patterns, the optimal allocation of UPRS, and factors influencing the allocation and utilization of recreational land (Gao, 2009; Liu and Wang, 2009). In landscape architecture, researchers have often discussed issues such as the services, facilities, and landscape planning of UPRS (Chen et al., 2016b); the coordination of architectural styles and urban 
appearance; and construction and landscaping (Guo et al., 2002; Chen, 2003). Urban planning researchers have focused on the reform and optimization of the UPRS system and the effects of UPRS in urban spatial planning (Yu and Zhang, 2015). Ecologists have concentrated on the impact of recreational activities on animals, plants, and the ecological environment.

Moreover, scholars from many other disciplines, such as sociology, management, culture, and economics, have analyzed the theoretical principles and planning strategies of UPRS (Wu, 1995; Ma, 2005) and evaluated UPRS safety (Chen et al., 2010). Besides, Yang Jianzhao et al. (2018) put forward a development strategy for urban recreational space system based on the "symbiotic theory". Through the inclusion of perspectives from different disciplines in the above discussions, UPRS research has become more thorough and specific.

\subsubsection{Impacts of UPRS development}

Along with rapid urbanization and the improvement of residents' quality of life in China, the planning and layout of UPRS have a great influence on the urban social culture, ecological environment, spatial structure, and urban landscape, all of which affect the stability and order of urban functions and operations.

To date, there have been many studies on the impacts of UPRS development outside China. Studies have focused on topics such as the influence of UPRS on neighborhood racial composition and neighborhood poverty (Duncan et al., 2013), the importance of UPRS for promoting social justice (Pryor and Outley, 2014), the effect of UPRS for improving symptoms among elderly Chinese patients with dementia ( $\mathrm{Li}$ and $\mathrm{Li}, 2017 \mathrm{a}$ ), and the impact of UPRS on the urban ecological environment (Moshiri and Donyamali, 2012). However, the number and depth of such studies focusing on contexts in China are insufficient. Some studies have examined the relationship between UPRS and urban residents' happiness (Gu, 2011) and the impact of urban recreational business districts (RBD) on urban development in China (Gu and Bao, 2002).

UPRS development has been shown to have both positive and negative effects. It is important for scholars to understand the overall relationship between the various functional elements of urban development and to discover imbalances and contradictions in this development to guide UPRS planning and optimize urban function. This will help to build harmonious, orderly, and livable cities.

\subsubsection{Spatio-temporal evolution laws and mechanisms of UPRS}

The study of spatio-temporal evolution laws and mechanisms of UPRS can enable the prediction of future evolution trends and provide theoretical support for the construction and development of UPRS.

To date, scholars have mainly used case studies to analyze the characteristics, regularity, dynamic mechanisms, and patterns of the evolution of UPRS for the purpose of further understanding UPRS (He, 2014; Lin, 2016). These analyses have mostly consisted of two dimensions. First, from the space perspective, the rules of UPRS evolution in most cities include not only the general, regionally differentiated rules, but also the gradually changing characteristics of recreational spaces in urban and suburban areas (Feng, 2009). Second, from the time perspective, because UPRS is a place for residents' recreational activities, the increase and diversification of residents' demands for recreation lead to the emergence of 
various types of UPRS with different features and the transformation of the service object of recreational space from private to public (Table 3).

Table 3 Evolution characteristics of urban public recreational space in China

\begin{tabular}{|c|c|c|}
\hline $\begin{array}{l}\text { Economic devel- } \\
\text { opment stage }\end{array}$ & $\begin{array}{l}\text { Production mode and lifestyle of } \\
\text { residents }\end{array}$ & $\begin{array}{l}\text { Characteristics of urban public } \\
\text { recreational space in China }\end{array}$ \\
\hline $\begin{array}{l}\text { Agrarian } \\
\text { economy era }\end{array}$ & $\begin{array}{l}\text { Most the society's wealth was } \\
\text { concentrated in the hands of a few } \\
\text { members of the ruling classes, and } \\
\text { the vast majority of people lived a } \\
\text { lifestyle of farming and } \\
\text { self-sufficiency. }\end{array}$ & $\begin{array}{l}\text { Recreational spaces were mainly owned by the ruling } \\
\text { classes, and urban recreational spaces were closed. Dur- } \\
\text { ing this period, there was little UPRS. Landscape gardens } \\
\text { in temples and suburbs that were not classified as royal or } \\
\text { noble became the main places for people to enjoy their } \\
\text { recreational time. }\end{array}$ \\
\hline $\begin{array}{l}\text { Industrial } \\
\text { economy era }\end{array}$ & $\begin{array}{l}\text { The basic purpose of the society } \\
\text { was the excessive pursuit of eco- } \\
\text { nomic benefits. People were eager } \\
\text { for various recreational activities } \\
\text { because of overwork. }\end{array}$ & $\begin{array}{l}\text { During this period, because of the development of social- } \\
\text { ist democratic politics, people were freed from the shack- } \\
\text { les of autocracy, and a large number of recreational } \\
\text { spaces that had been the private property of feudal lords } \\
\text { were gradually opened to the public. Because of the rapid } \\
\text { growth of the population in China and the impact of urban } \\
\text { expansion, social spaces in neighborhoods had been re- } \\
\text { placed by high-rise buildings. As people's desire for } \\
\text { communication increased, potential and practical recrea- } \\
\text { tional demands prompted cities to build a large amount of } \\
\text { UPRS, such as museums and commercial streets. }\end{array}$ \\
\hline $\begin{array}{l}\text { Experience } \\
\text { economy era }\end{array}$ & $\begin{array}{l}\text { With improvements in people's } \\
\text { living standards and changes in } \\
\text { cognition, people's recreational } \\
\text { demands are gradually diversified } \\
\text { and personalized. }\end{array}$ & $\begin{array}{l}\text { With changes in people's recreational demands, city } \\
\text { development has gradually changed from aiming only to } \\
\text { create recreational space to aspiring to construct UPRS } \\
\text { that can meet the recreational needs of different classes of } \\
\text { people. }\end{array}$ \\
\hline
\end{tabular}

Additional study in this area has found that different types of UPRS have distinct spatio-temporal evolution rules. Previous work beginning with analyses of historical Recreational Business District (RBD) forms have proposed three evolution models: the central business district model (e.g. Central District, Hong Kong) (Bao and Gu, 1998; Liu, 2003; Tao and Li, 2005), the new town center model (e.g. Tianhe, Guangzhou) (Bao and Gu, 2002), and the commercial street model (Chunxi Road, Chengdu). Other studies have analyzed the evolution of the RBD space shape in the information age, finding that the RBD evolution trends were multi-core, multilayered, and networked (Chen and $\mathrm{Wu}, 2007$ ). The evolution of recreational belts around metropolises has been found to have undergone three stages of growth: pole evolution, point-axis evolution, and network evolution ( $\mathrm{Wu}$ and $\mathrm{Wu}, 2005$ ). Other studies have analyzed the evolution characteristics of the Shanghai recreational belt around the metropolis, finding that the evolution process has changed from dispersion to agglomeration, and the agglomeration shapes have evolved from scattered spots to growth poles, axes and eventually blocks (Dang et al., 2017).

\subsubsection{Space imagery features of UPRS}

Using space imagery to analyze spatial structures is one of the methods used in spatial structure studies. Examining space imagery features is a popular way to illustrate perceptions of the surrounding environment, and this approach has attracted many scholars from the discipline of urban geography, especially urban social geography (Gu and Song, 2001). Recreationists' perceptions can influence use patterns and inform the planning and manage- 
ment of UPRS.

The study of the space imagery features of UPRS is of great significance for city planning and construction with the goal of maximizing happiness, livability, and appropriate travel (Tian, 2013). Existing studies have taken urban residents and outside visitors as research subjects, seeking to analyze the UPRS imagery features through case studies using specific methods such as cognitive maps, imagery sketches, and questionnaire surveys (Yin et al., 2010; Lin and Tian, 2011; Tian, 2013). Inspired by urban imagery theory, Tian Fengjun (2010) proposed the concept of the "recreation image complex".

\subsubsection{Sustainable utilization of UPRS}

Understanding the use patterns and users of UPRS is necessary for effective planning and management (Chen et al., 2018). Along with the widely applied sustainable development theory, the planning and design of UPRS does not only need to meet residents' recreational needs, but also to integrate various types of land to provide UPRS in China, including urban industrial heritage sites, historic streets, abandoned industrial lands, residential green spaces, and sports fields (Zhang and Xia, 2008; Yi and Horton, 2015).

The key to the sustainable use of UPRS is coordination among the various elements (time, cost, and space for recreation), through a combination of material and socioeconomic planning (Manuel and Fred, 2004). Most existing studies are based on a systems perspective and consider the general elements of UPRS to include tourists seeking recreation, recreational resources, recreational services, and facilities (Liu, 2015). These studies have explored, for example, the integrative design of children's UPRS on a commercial pedestrian street (Li and $\mathrm{Li}, 2017 \mathrm{~b}$ ) and the integrative design of UPRS and urban transportation (Liang and Luo, 2009). Other scholars have been concerned about the reuse of UPRS, especially the post-competition utilization of Olympic venues ( $\mathrm{Hu}, 2006)$.

To date, studies on the sustainable use of UPRS have some problems in China. The core issue is the lack of studies on integration mechanisms and approaches in the process of UPRS construction. Most highly cited articles on UPRS have focused on the specific traditional types of UPRS, such as recreational belts around metropolises, urban RBDs, urban parks, waterfront recreational spaces, and urban green spaces. There has been insufficient work analyzing UPRS systems overall. At the same time, with the development of UPRS and the constantly changing demands of recreationists, many new types of UPRS have recently emerged; these new types include libraries, art museums, science and technology museums, and sports venues ( $\mathrm{Lu}, 2013)$. However, to date, there has been a relatively limited number of studies on these new UPRS types in China.

\subsubsection{Governmental decision making on UPRS}

UPRS is an integral component of livable cities. These spaces play a critical role in how citizens spend their spare time. Rapid urbanization and spontaneous socioeconomic development have brought about profound change in residents' recreational time and in per capita disposable income. Recreational activities have become an important way for people to alleviate pressure in their lives, and providing sufficient UPRS to meet citizens' increasing needs is the responsibility of policy makers. Because of the increasingly diversified needs for recreation, UPRS provisioning has transformed from a public process dominated by the 
government to a multiple provision mode with various suppliers. This has created a system of recreation provision with the central government offering public recreational services in national parks, the city government providing urban recreational opportunities, the public sector maintaining basic recreational services, and the private sector providing high-end, diversified recreational services (Zhang, 2010). Because UPRS is an essential component of public welfare, more humanistic concern for vulnerable groups is needed in the planning and design of these spaces. However, scholars have given little attention to this issue.

In the process of conducting this review, we noticed that UPRS has been associated with a number of government administrative departments (e.g. the Tourism Bureau, Forestry Bureau, Landscaping Bureau, Planning Bureau, Construction Bureau, Industry and Commerce Bureau, Cultural Bureau, Education Bureau, and Agricultural Bureau), and the management pattern of these spaces is characterized by fragmentation and multi-headed administration. Past work on UPRS has suggested four integrative schemes of government administrative departments based on the realistic conditions of cities: a) Cities driven by recreational tourism (Zhuhai and Lijiang) can manage the relevant functions under the Tourism Bureau; b) cities driven by commercial tourism (Beijing, Shanghai, and Shenzhen) can establish inter-ministerial consultation committees to be led by the Tourism Bureau; c) small and medium-sized cities where the tourism industry is undeveloped can manage related functions under the Cultural Bureau; and d) metropolises where the tourism industry is underdeveloped can establish inter-ministerial consultation committees led by the Cultural Bureau (Ye et al., 2015).

\subsection{Methodology and techniques in UPRS research in China}

Research on UPRS in China cannot be carried out in an orderly manner without the support of relevant technological methods. In the process of summarizing existing studies on UPRS in China, we found that the research methods used mainly consist of descriptive analysis, conceptually based approaches, models, or statistical methods (Dann et al., 1988). After China's UPRS research entered the growth period (2001-2018), examining the top 10 most highly cited articles each year, we found that the theoretical studies focused on descriptive and conceptually based methods, whereas the empirical studies focused on models and statistical methods. In these articles, statistical methods were the most frequently used (67.5\%); these studies analyzed the evolution of the structure of recreational space (Huang et al., 2010), the satisfaction of UPRS users (Xiao and Du, 2011; Lian et al., 2012; Mao et al., 2013), the spatial structure characteristics and distribution rules (Su et al., 2004; Shi and Pei, 2007; Liu et al., 2010b; Yang and Ma, 2015; Lin and Mao, 2017), and UPRS accessibility (Ren and Wan, 2016; Yang and Shi, 2016) using spatial metrology, remote sensing image processing, and other technical tools, with software packages such as AutoCAD, SPSS, and ArcGIS. Articles focusing on models (21.3\%) mainly conducted analyses of the spatial system structure of UPRS (spatial discrete index method), recreational value evaluation (recreationists' perception distance), UPRS accessibility evaluation (network accessibility index), and comprehensive assessment of tourism destinations (spatial data overlay). Conceptual approaches (8.1\%) were used widely in basic theoretical research on UPRS (Wu et al., 2003), including analyses of the concept and its connotations, type division, components of the 
UPRS system, and examinations of the driving mechanisms. Descriptive methods (3.1\%) were mainly used in describing the UPRS development process (Chen, 2013), related literature reviews (Feng, 2006a; 2006b; Li et al., 2014; Zhu et al., 2014), and summaries of and comments on non-Chinese UPRS planning and design experiences (Wu, 1995; Fang and $\mathrm{Wu}$, 2012; 2015; Yu and Zhang, 2015) (Table 4).

Table 4 Main application trends of methods in urban public recreational space research in China (2001-2018)

\begin{tabular}{llc}
\hline Research method & \multicolumn{1}{c}{ Main application trends } & ${\text { Percentage }(\%)^{\mathrm{a}}}^{-}$ \\
\hline Descriptive & $\begin{array}{l}\text { Literature review, comments on non-Chinese urban public recreational space } \\
\text { planning and design experiences }\end{array}$ & 3.35 \\
Conceptually & $\begin{array}{l}\text { Basic theoretical research, including the analysis of the concept of UPRS } \\
\text { and its connotations, type division, the components of the UPRS system, and }\end{array}$ & 8.94 \\
based & driving mechanisms & 21.23 \\
Models & $\begin{array}{l}\text { Ideal structural pattern of UPRS, recreational value evaluation, comprehen- } \\
\text { sive assessment of tourism destinations }\end{array}$ & \\
Statistics & $\begin{array}{l}\text { The evolution of recreational space structure, satisfaction of UPRS users, spatial } \\
\text { structure characteristics and distribution rules, accessibility of UPRS }\end{array}$ & 66.48 \\
\hline
\end{tabular}

${ }^{a}$ Percentage of the top 10 articles cited each year from 2001 to 2018 (179 papers in total) on each specific research method

GIS deserves special mention as a widely used technology in the disciplines of planning, geography, environment, ecology, and tourism that is also often used in UPRS research. The earliest work applying GIS spatial analysis techniques in recreation research appeared in the United States in the late 1960s (Luo et al., 2006). After GIS technology was introduced in China, it was soon applied to UPRS research. Supported by GIS software, a variety of UPRS research has incorporated network analysis, spatial analysis, digital elevation analysis, and geographical statistics. These studies have mainly examined the connectivity and accessibility of UPRS, recreationists' perceptions of distance, the distribution of recreational resources, the locations of UPRS, and spatial distribution characteristics. Hodges and Carlton (1972) used Synagraphic mapping tools to analyze UPRS locations and calculate the service radius of urban recreational centers.

\subsection{The spatial scale of UPRS research in China}

Aiming to explore issues, objectives, and approaches, UPRS research in China has been carried out at several separate levels, including state, regional (e.g. urban agglomeration, or province), city (e.g. Shanghai or Beijing), and within-UPRS levels. At the state level, studies have taken a macro perspective, focusing on the overall environment, service quality, policy analysis, and regional differences in UPRS, as well as China's UPRS development strategies, upgrade plans, and future directions. The topics at the regional level are similar to those at the national level and mainly include development characteristics, facilities evaluation, environmental optimization, and development strategies for UPRS in different regions. Urban agglomeration, in particular, has attracted the attention of many scholars focusing on China at this level. Urban agglomeration is an ideal area for the development of tourism industry clusters and the centralized location of UPRS because of factors such as market size, convenient travel, and abundant land (Liu and Liang, 2015).

The city is the most common scale for UPRS studies. Research at this level began with 
categorizing the type and quantity of UPRS. The next step was the analysis of the morphological structure and distribution regularity of different types of UPRS. Studies have also comprehensively evaluated the quality of the urban recreational environment, using data on the urban population size, the recreational atmosphere, the natural environment, and other related characteristics. For targeted analysis, scholars have often chosen specific types of UPRS or UPRS considered "typical," such as recreational belts around the metropolises (Wu, 2001; Su et al., 2004; Li et al., 2010a), urban recreational business districts (Bao and Gu, 2002; Gu and Bao, 2002; Yu and He, 2003; Bian and Zhang, 2004; Tao and Huang, 2006; Chen and Bao, 2012; Zhu et al., 2015; 2017), urban parks (Liu et al., 2010a; Mao et al., 2012; Mao et al., 2013; Tao et al., 2013; Feng et al., 2014; Chen et al., 2016a; Wang and Liu, 2017; Wang et al., 2017b; Xiao et al., 2017; Liang and Zhang, 2018), urban green recreational spaces (Zhang et al., 2013; Jim and Chen, 2006), waterfront recreational spaces (Wu and Jia, 2002; Zhang et al., 2011), suburban recreational spaces (Wang, 2003; Liu et al., 2010b; Liu and $\mathrm{Mu}, 2016$ ), ecological recreational spaces (Li, 2014; Sun et al., 2016a; 2016b; Yang et al., 2018), or theme parks (Bao, 1994; 1997; Lian et al., 2012; Cheng et al., 2016; Yan et al., 2016).

There have also been studies on UPRS features on multiple dimensions from the perspective of multi-scale fusion and nesting patterns. With the socioeconomic development of small and medium-sized cities, the exploration and development of UPRS has attracted the participation of multiple stakeholders, including the Urban Planning Department, academics, and residents. Studies on UPRS have also branched out from a focus on large cities such as Beijing and Shanghai to examine small and medium-sized cities. Research about UPRS in small and medium-sized cities has focused on topics such as planning and design, the creation of a recreational atmosphere, the improvement of the quality of recreational experiences, and public administration policies.

\section{Conclusions, prospects and limitations}

\subsection{Conclusions}

Based on articles published on UPRS from the China National Knowledge Internet and the Web of Science databases, the present study systematically reviewed and discussed the main features and contents of UPRS research in China from 1985 to 2018, as well as addressing the research methods and spatial research scales employed.

(1) Overall, studies on UPRS in China show a growing scope and depth. Since 1985, scholars have carried out systematic research from different perspectives, at multiple spatial levels, and drawing on multiple methods. This work has yielded many enlightening research findings.

(2) The main characteristics of UPRS research in China identified in the present study included phase characteristics and hierarchical structure characteristics. We divided the research phases into the starting period (1985-2000), the growth period (2001-2009), and the consolidation period (2010-2018). Our presentation of the hierarchical structure features was accomplished using a "stars holding the moon" visual illustration of the knowledge network. In this presentation, "recreational space" was the core, surrounded by multiple re- 
search issues.

(3) The topics covered by UPRS research in China show a trend toward interdisciplinarity and diversified development. Since 1985, Chinese scholars have conducted a great deal of empirical research in this area, including examinations of planning practices. This previous work has made critical contributions to China's macro-level strategy, regional development at different levels, and UPRS planning and development. To some extent, China has achieved an application of "theory guiding practice" and "practice promoting theory," through the mutual promotion of research and practice. The main topics addressed by UPRS research in China can be summarized by nine aspects: the classification of UPRS, spatial structure and layout, comprehensive evaluation, planning and development, the impacts of UPRS development, spatio-temporal evolution laws and mechanisms, space imagery features, sustainable utilization, and governmental decision making.

(4) The research methods used in UPRS research in China have changed from qualitative to primarily quantitative methods. The specific methods that have been employed include descriptive analyses, conceptually based approaches, models, and statistics, with statistics being the most frequently applied (67.5\%), descriptive analyses being the least commonly used (3.1\%), and models and conceptual-based approaches accounting for $21.3 \%$ and $8.1 \%$ of the examined studies, respectively.

(5) The spatial scale of the study is moving from the micro level to the macro level. Although the micro level remains the main spatial scale for UPRS research in China, studies on the state and region are increasing.

\subsection{Future priorities and directions for UPRS research in China}

In response to the problems and deficiencies identified in existing research, those conducting future work on UPRS in China should follow these recommendations:

(1) Future studies of UPRS in China should pay more attention to basic theoretical research and the expansion and deepening of the research content. Utilization of UPRS in Western cities has always been considered a basic right of city residents. This understanding has a profound social value basis that is clearly reflected in urban planning and design. Likewise, the theoretical approach to UPRS planning and development in Western contexts is systematic and mature, and related planning practices are relatively abundant in these contexts. However, in China, urban public space, especially UPRS, is a relatively new concept, and its research foundation is still weak. The study of UPRS involves multiple disciplines, such as urban planning, geography, recreational management, and landscape architecture. Input from the disciplines of sociology, culture, and ecology to research theory and practice in China currently lags behind the situation in Western countries.

In response to residents' urgent need for UPRS, and to guide the practice of UPRS planning and management during the process of rapid urbanization, we need to translate and adapt UPRS theories originating in Western studies to account for Chinese characteristics. This should include the basic concept of UPRS, as well as its connotations and classification systems. Promoting interdisciplinary and innovative research methods can help to build UPRS theory and to expand the content of research on UPRS in China. Although studies on UPRS have pointed to cross-disciplinary connections, the links between related studies are 
not entirely clear. Future research efforts ought to enhance cooperation among various disciplines, such as tourism geography, urban planning, ecology, psychology, sociology, economic management, landscape architecture, and mathematical statistics. In this process, we should prioritize using innovative research methods, seeking new entry points, establishing a long-term database on UPRS from a multidimensional spatial hierarchy perspective, deepening the scope of empirical research based on primary data, and making full use of space-related socioeconomic statistical data in our research.

The infusion of humanistic concepts and categories is a popular trend in international UPRS research. Human needs, objectives, and benefits are the basis for the formation of UPRS. Research on UPRS in China should not only provide top-down, macro-level analyses of UPRS strategies; it should also include bottom-up, in-depth explorations of multiple subjects, including different classes, genders, and subcultures. Therefore, it is essential that researchers appreciate and examine the humanistic background behind UPRS phenomena from a philosophical perspective.

(2) This work should enhance the integration of UPRS in multiple spatial hierarchies and stress the importance of the spatial research scale. Spatial scale is the basis of UPRS research, and the spatial distribution of UPRS at state, regional, city, and within-UPRS levels ought to be further discussed, emphasizing the participation of governments, companies, and scholars at different levels.

At the level of the nation and region, with the advancement of China's new urbanization process, the planning and construction of UPRS in small and medium-sized cities are quite different from these processes in large cities. Therefore, it is necessary to research UPRS in small and medium-sized cities, specifically. The development rules for UPRS also differ significantly across regions, interactively influenced by various factors such as the natural environment, economic development, socio-cultural features, and macro policies. It is necessary to comparatively analyze the differences in UPRS progress in different regions to identify the factors and mechanisms so that practical resources can be provided for other similar geographical areas.

At the within-UPRS level, with the diversification of people's demands, there are individualized features in the utilization of UPRS. Compared with developed countries, China is rich in features such as urban industrial heritage sites, historic streets, abandoned industrial lands, residential green spaces, and sports fields, but the potential recreational value of these features has yet to be fully explored. The integration of various types of geographical areas will increase the amount and quality of UPRS, prompting an increase in the use of the established and future UPRS in China. An example of this type of integration is the Beijing 798 Art District, which is a typical case of the integration of an industrial heritage conservation area into a UPRS system. Research at the within-UPRS level needs to be deepened, further discussing issues including the complementary patterns and rational layout of different types of UPRS (e.g. urban parks, theme parks, recreational belts around metropolises, and RBDs) to contribute to optimizing the spatial layout and maximizing the recreational value of UPRS.

Although the academic community has made some progress in the study of UPRS from the perspective of two- and three-dimensional spatial hierarchies, more needs to be done in 
this research area. It is necessary to strengthen the synthesis of research at various levels of UPRS, including the links between different types of physical UPRS. The integration of physical and socio-economic UPRS, and the integration of physical UPRS with recreational space and spaces of capital and power will allow UPRS research to form a complete system to promote UPRS sustainable development.

(3) It is necessary to strengthen the operation and management of UPRS and attend to the relationship between urban space and UPRS. The essence of UPRS management is the construction and management of a recreational culture and a recreational environment (Feng, 2006a). To realize the scientifically informed management of UPRS, it is necessary to strengthen related research such as work on investment, financing methods, capital operations, human resource management, relevant departments working in harmony with residents, evaluation systems before and after the construction of UPRS, the discovery and reproduction of recreational culture, the protection of recreation resources, and the organization and marketing of the recreational product.

In addition, UPRS is an organic part of urban space, and it is necessary to strengthen the comprehensive management of UPRS, optimize the location and spatial coupling of UPRS with urban space, and analyze the coupling mechanism and methods from macro, mezzo, and micro hierarchical levels.

(4) We should conduct research on UPRS in China from a globalization perspective and construct a new research system that considers the characteristics of the Chinese context. UPRS is a hallmark product of modernization and globalization (Shen, 2011). Along with the diversification of economic industry and globalization, people's lifestyles will turn increasingly toward diversification and individuation. At the same time, information exchanges will speed up around the world, promoting mutual learning between cities. In terms of UPRS, international planning experiences will provide useful references for UPRS planning and design in China. This process will help with the development of more UPRS that can satisfy the diversified recreational demands of people of different ages and from different cultural backgrounds.

Although international UPRS research can provide a starting point for China's UPRS planning, UPRS planning and development in China need to be adapted to the specific conditions of the Chinese context, highlighting the recreational behavioral characteristics of China's residents and taking into account differences in recreational demands across the country's regions. Based on the national conditions in China, Chinese government and relevant departments should formulate specific policies and systems for UPRS planning and establish a complete management system and policy guarantee system for UPRS.

However, national standards guiding the construction of UPRS in China are currently insufficient. It is necessary to set up a more detailed planning system and a standardized economic index that is adapted to China's national conditions, aimed at different types of UPRS and different planning levels (e.g. overall planning, regulatory detailed planning, constructive detailed planning, or landscape design), and to build a Chinese UPRS management system with a global vision. This could provide a policy basis and scientific support for the construction and management of UPRS in China. 


\subsection{The limitations of the study}

This study has two main limitations in terms of research scope, which indicate the need for further research. Firstly, this study only examined and analyzed the studies of UPRS in China from a macro perspective. It may neglect studies on individualized and innovative UPRS. It may also ignore the differences in UPRS research caused by the regional development differences. Secondly, the method of data collection also has its limitations. For example, this study only looked at articles published on the Web of Science and China National Knowledge Internet databases. The literature search keywords of "recreational space in China" may lead to insufficient understanding of the research contents. Therefore, the data collection for UPRS studies should be more comprehensive and abundant in future research. Comparative studies at different research levels of UPRS in different development areas should also be addressed.

\section{References}

Bao Jigang, 1994. A study on the distribution of theme parks. Geographical Research, 13(3): 83-89. (in Chinese)

Bao Jigang, 1997. A systematic analysis of the influential factors to theme park development. Acta Geographica Sinica, 52(3): 47-55. (in Chinese)

Bao Jigang, Gu Shiyun, 1998. Tentative research on RBD of city. Planners, 14(4): 59-64, 126. (in Chinese)

Bao Jigang, Gu Shiyun, 2002. The formation and development of recreational business district in Guangzhou city, China. Human Geography, 17(5): 1-6. (in Chinese)

Bian Xianhong, Zhang Shufu, 2004. Research on the exploration and development of urban recreational business district. Economic Geography, 24(2): 206-211. (in Chinese)

Chen Bixia, Qi Xinhua, Qiu Zhenmian, 2018. Recreational use of urban forest parks: A case study in Fuzhou National Forest Park, China. Journal of Forest Research, 23(3): 183-189.

Chen Chunhua, 2003. Lively design of urban open leisure space. Journal of Chongqing Jianzhu University, 25(2): 5-8. (in Chinese)

Chen Hongtao, $\mathrm{Wu}$ Lian, 2007. The evolution of unban recreation business areas in China in infomation age. Journal of Northwest University (Natural Science Edition), 37(5): 835-838. (in Chinese)

Chen Qiuxiao, Hou Yan, Wu Shuang, 2016a. Assessment of accessibility to urban parks in Shaoxing City from the perspective of opportunity equity. Scientia Geographica Sinica, 36(3): 375-383. (in Chinese)

Chen Weizhi, Fang Jie, Huang Lijie, 2010. A study on the safety comprehensive assessment system of children's outdoor amusement and rest space in the residential areas. Journal of Zhejiang Sci-Tech University, 27(5): 768-772. (in Chinese)

Chen Yiyong, Liu Tao, Xie Xiaohuan et al., 2016b. What attracts people to visit community open spaces? A case study of the Overseas Chinese Town community in Shenzhen, China. International Journal of Environmental Research and Public Health, 13(7): 644.

Chen Yu, 2013. The evolution and style of urban recreation space. Chinese Landscape Architecture, (2): 69-72. (in Chinese)

Chen Zhigang, Bao Jigang, 2012. The spatial morphological evolution of RBD and its determining mechanism in a typical scenic tourist city: The case study of Yangshuo County, China. Geographical Research, 31(7): 1339-1351. (in Chinese)

Cheng Qian, Guo Jingjing, Ling Supei, 2016. Fuzzy importance-performance analysis of visitor satisfaction for theme park: The case of Fantawild Adventure in Taiwan, China. Current Issues in Tourism, 19(9): 895-912.

Dang Ning, Wu Bihu, Yu Qinhui, 2017. The spatiotemporal evolution and dynamic mechanism of the Shanghai ReBAM from 1970 to 2015. Tourism Tribune, 37(2): 281-291. (in Chinese)

Dann G, Nash D, Pearce P, 1988. Methodology in tourism research. Annals of tourism research, 15(1): 1-28. 
Dong Nannan, Chen Yixuan, Zhang Shenghong, 2015. Intergeneration transformation of park recreation of children in downtown Shanghai. Chinese Landscape Architecture, (9): 38-42. (in Chinese)

Duncan D T, Kawachi I, White K et al., 2013. The geography of recreational open space: Influence of neighborhood racial composition and neighborhood poverty. Journal of Urban Health, 90(4): 618-631.

Fang Jia, Wu Chengzhao, 2012. Analysis of the process of urban open space planning approaches in USA. Chinese Landscape Architecture, (11): 62-67. (in Chinese)

Fang Jia, Wu Chengzhao, 2015. Contents and case study of open space planning in the USA. Foreign Planning Studies, 39(5): 76-82. (in Chinese)

Feng Weibo, 2006a. The progress of urban recreation space research and its major development field in China. Advances in Earth Science, 21(6): 585-592. (in Chinese)

Feng Weibo, 2006b. On the progress of urban recreation space research in China: Statistic analysis based on the relevant academic documents. Economic Geography, 26(Suppl.2): 21-23. (in Chinese)

Feng Weibo, 2009. Urban Recreational Space Analysis and Integration. Beijing: Science Press. (in Chinese)

Feng Weibo, 2010. The structural pattern of urban recreation space system. Architectural Journal, (Suppl.2): 150-153. (in Chinese)

Feng Weibo, Long Bin, Zhang Shulin, 2009. Evaluation of urban recreation space quality based on perception of tourist in Chongqing metropolis. Human Geography, 24(6): 91-96. (in Chinese)

Feng Yueyi, Hu Tangao, Zhang Lixiao, 2014. Impacts of structure characteristics on the thermal environment effect of city parks. Acta Ecologica Sinica, 34(12): 3179-3187. (in Chinese)

Gan Rui, 2004. Analysis and appraisal on recreational environment in metropolis in China. Human Geography, 19(3): 93-96. (in Chinese)

Gao Yongshan, 2009. Study on the special distribution in recreational belt around metropolis of Qingdao, China. Journal of Dezhou University, 25(2): 81-84. (in Chinese)

Gu Chaolin, Song Guochen, 2001. Urban image space and main factors in Beijing. Acta Geographic Sinica, 56(1): 64-74. (in Chinese)

Gu Shiyun, Bao Jigang, 2002. The impacts of RBD on urban development in Guangzhou City, China. Scientia Geographica Sinica, 22(4): 489-494. (in Chinese)

$\mathrm{Gu}$ Weijie, 2011. Analysis on relationship between public recreational space construction of Beijing and residential happiness index. Journal of Sports and Science, 32(3): 43-48. (in Chinese)

Gu Xiaokun, Tao Siyuan, Dai Bing, 2017. Spatial accessibility of country parks in Shanghai, China. Urban Forestry \& Urban Greening, 27: 373-382.

Guo Xu, Guo Enzhang, Lu Fei, 2002. Construction of high quality recreation space in cities. Journal of Harbin University of C. E. \& Architecture, 35(3): 84-91. (in Chinese)

He Feng, 2014. Study on the spatial extension mechanism of the sports recreation in Shanghai from the perspective of public service equalization. Sport Science Research, 35(5): 48-56. (in Chinese)

Hodges Louis, Carlton S. Van Doren, 1972. Synagraphic mapping as a tool in locating and evaluating the spatial distribution of municipal recreation facilities. Journal of Leisure Research, 4(4): 341-353.

$\mathrm{Hu}$ Hao, 2006. Summary and comments: Modern Olympic Games on expansion of public recreation spaces in host cities. Social Science of Beijing, (5): 30-33. (in Chinese)

Huang Nanyuan, He Fangzi, 1979. Brief introduction to "Taipei Land Use Zoning Control Bill”. Urban and Plan, 5(1): 15-22. (in Chinese)

Huang Tai, Bao Jigang, Dai Xuejun, 2009. Fractal study on spatial structure of city recreation sites system: A case study of Suzhou City Area, China. Progress in Geography, 28(5): 735-743. (in Chinese)

Huang Wei, Jiang Liao, Gao Xin, 2010. The spatial structure evolution and development model about recreational area in Chongqing Bishan. Economic Geography, 30(1): 167-172. (in Chinese)

Jiao Limin, Liu Yaolin, 2010. Geographic field model based hedonic valuation of urban open spaces in Wuhan, China. Landscape and Urban Planning, 98(1): 47-55.

Jim C Y, Chen Wendy Y, 2006. Recreation-amenity use and contingent valuation of urban greenspaces in Guangzhou, China. Landscape and Urban Planning, 75(1/2): 81-96. 
Li Dongmei, Li Xiaoxue, 2017. The effect of folk recreation program in improving symptoms: A study of Chinese elder dementia patients. International Journal of Geriatric Psychiatry, 32(8): 901-908.

Li Hua, 2014. The pattern and optimization of urban ecological recreation space in Shanghai, China. Economic Geography, 34(1): 174-180. (in Chinese)

Li Le, Liu Jiaming, Song Tao et al., 2014. Research progress of urban green belt and recreational use. Progress in Geography, 33(9): 1252-1261. (in Chinese)

Li Lulu, Li Min, 2017. A research on the integrated design of children's recreational site in commercial pedestrian street. Design, (5): 18-19. (in Chinese)

Li Renjie, Guo Fenghua, An Ying, 2011. The structure of recreational space types and spatial pattern around Beijing metropolis in recent 10 years. Human Geography, 26(1): 118-122. (in Chinese)

Li Renjie, Yang Ziying, Sun Guiping et al., 2010a. The evaluation system and methods designed for estimating the developing maturation of recreation belt around metropolises. Geographical Research, 29(8): 1416-1426. (in Chinese)

Li Xiaoma, Liu Changfu, 2009. Accessibility and service of Shenyang's urban parks by network analysis. Acta Ecologica Sinica, 29(3): 1554-1562. (in Chinese)

Li Yuefeng, Li Junmei, Fei Yu et al., 2010b. An assessment on the influence of cherry blossom on recreation value of Kunming Zoo with travel cost method. Yunnan Geographic Environment Research, 22(1): 88-93. (in Chinese)

Lian Tonghui, Yu Caihua, Bao Xianjian et al., 2012. Research on the satisfication of theme park visitors based on fuzzy comprehension evaluation: A case study in Wuhu Fantawild Adventure. Resources Science, 34(5): 973-980. (in Chinese)

Liang Huilin, Zhang Qingping, 2018. Assessing the public transport service to urban parks on the basis of spatial accessibility for citizens in the compact megacity of Shanghai, China. Urban Studies, 55(9): 1983-1999.

Liang Zhichao, Luo Jianhe, 2009. Integrative design and research of recreation space and urban transportation: A case study of the waterfront recreation spaces in the old towns in Pearl River Delta Area. South Architecture, (5): 45-47. (in Chinese)

Lin Qing, Tian Fengjun, 2011. Empirical study of the urban recreational space of city's image building: A case study on Nanchang City. Tourism Forum, 4(4): 90-94. (in Chinese)

Lin Yan, Mao Jiangxing, 2017. Nanning ReBAM spatial distribution situation and optimization research. Journal of Guangxi Teachers Education University (Natural Science Edition), 34(2): 115-121. (in Chinese)

Lin Zhanglin, 2016. Spatial-temporal evolution mode of Shanghai city tourism and leisure public space. Tourism Science, 30(2): 79-94. (in Chinese)

Liu Changfu, Li Xiaoma, Han Dong, 2010a. Accessibility analysis of urban parks: Methods and key issues. Acta Ecologica Sinica, 30(19): 5381-5390. (in Chinese)

Liu Hongming, Mu Ye, 2016b. Design and research of urban suburban recreation space. Journal of Jilin Jianzhu University, 33(2): 67-70. (in Chinese)

Liu Jiaming, Wang Run, 2009. Suggestions for the allocation and management of recreational land in Beijing city: A perspective based on Hong Kong and Singapore as cases. Human Geography, 24(2): 107-111. (in Chinese)

Liu Jiaming, Wang Run, Chen Tian, 2010b. Factors of spatial distribution of recreation areas in peri-urban Beijing, Journal of Geographical Sciences, 20(5): 741-756.

Liu Shaohe, Liang Mingzhu, 2015. Development path of tourism agglomeration in recreation belt around urban agglomeration of the Greater Pearl River Delta: A case study of tourism section for coastal region and mountain area in Guangdong Province. Economic Geography, 35(6): 190-197. (in Chinese)

Liu Shuang, 2015. Study on the integration path of tourism urban recreational space based on system perspective. Heihe Journal, (8): 10-11. (in Chinese)

Liu Songling, 2003. From CBD to RBD: Explore the trend of traditional CBD development. Urban Research, 18(4): 59-64. (in Chinese)

Lu Zhouxiang, 2013. From Hongkew Recreation Ground to Bird's Nest: The past, present and future of large sports venues in China. The International Journal of the History of Sport, 30(4): 422-442. 
Luo Yanju, Bi Hua, Zhao Zhizhong et al., 2006. Review of GIS applications to outdoor recreation management and planning. Journal of Anyang Institute of Technology, 6(1): 48-54. (in Chinese)

Ma Huidi, 2005. A glance on urban planning for recreational ground in Western countries. Qilu Journal, (6): 147-153. (in Chinese)

Manuel Baud-Bovy, Fred Lawson, 2004. Tourism and Recreation: Handbook of Planning and Design. Butterworth-Heinemann Ltd.

Mao Xiaogang, Song Jinping, Feng Huihui et al., 2013. Residents recreation satisfaction index of Beijing city parks based on SEM. Geographical Research, 32(1): 166-178. (in Chinese)

Mao Xiaogang, Song Jinping, Yang Hongyan et al., 2012. Changes of the spatial pattern of Beijing city parks from 2000 to 2010. Progress in Geography, 31(10): 1295-1306. (in Chinese)

Moshiri S R, Donyamali A, 2012. Environmental impact assessment of large recreational, sports, and cultural complexes on urban spaces case study: Hezar O Yek Shahr Recreational, Sports, and Cultural Complex District 22 of Tehran Municipality. Life Science Journal, 9(4): 131-153.

Pryor B N K, Outley C W, 2014. Just spaces: Urban recreation centers as sites for social justice youth development. Journal of Leisure Research, 46(3): 272.

Qin Xue, 2003. Systematic study on urban recreational spatial structure: A case study of Ningbo city, China. Economic Geography, 23(2): 267-271. (in Chinese)

Ren Lili, Wan Qingxu, 2016. Accessibility analysis of outdoor public recreational space of Qingdao, China. Geospatial Information, 14(7): 78-81. (in Chinese)

Shen Hao, 2011. Analysis of the impact of recreation development on urban space. Urban Construction, (1): 8-8. (in Chinese)

Shi Qianyou, Pei Bo, 2007. A research of attraction spatial characteristics in the recreational belt around Xi'an city. Journal of Shaanxi Normal University (Natural Science Edition), 35(4): 102-106. (in Chinese)

Su Ping, Dang Ning, Wu Bihu, 2004. Attraction categories and spatial characteristics in recreational belt around metropolis: Beijing case study. Geographical Research, 23(3): 403-410. (in Chinese)

Sun Kun, Tang Chengcai, Zhong Linsheng, 2016a. Siting of urban recreational eco-space based on population characteristics: A case study of Changshu City, China. Progress in Geography, 35(6): 714-723. (in Chinese)

Sun Kun, Zhong Linsheng, Zhang Aiping et al., 2016b. Comparative analysis on the leisure values of urban ecological recreation spaces: A case study of Changshu city, China. Geographical Research, 35(2): 256-270. (in Chinese)

Tao Wei, Huang Rongqing, 2006. Urban recreation business district and its relative factors: A case study of Guangzhou, China. Human Geography, 20(3): 10-13. (in Chinese)

Tao Wei, Li Limei, 2005. Spatial structure evolution mode of urban recreational business district in Hong Kong. Planning Progress in Hong Kong, (6): 69-75. (in Chinese)

Tao Xiaoli, Chen Mingxing, Zhang Wenzhong et al., 2013. Classification and its relationship with the functional analysis of urban parks: Taking Beijing as an example. Geographical Research, 32(10): 1964-1976. (in Chinese)

Tian Fengjun, 2010. A study on the building of urban recreation space image: Based on the concept of recreation image complex. Journal of Business Economics, (11): 91-96. (in Chinese)

Tian Fengjun, 2013. City recreation space imagery features of Nanchang City. Resources Science, 35(5): 1095-1103. (in Chinese)

Wang Degen, Sun Feng, Liu Changxue et al., 2017a. Impact of low-carbon public transport system on urban recreational spatial: A case study of center city of Suzhou. Tourism Tribune, 32(10): 78-90. (in Chinese)

Wang Kai, Liu Jianjun, 2017. The spatiotemporal trend of city parks in Mainland China between 1981 and 2014: Implications for the promotion of leisure time physical activity and planning. International Journal of Environmental Research and Public Health, 14(10): 1150.

Wang Xuhui, Liu Kewei, Wang Kai et al., 2017b. Spatiotemporal dynamics of urban parks and their driving forces in Xi'an, China from1949 to 2015. Open House International, 42(3): 112-115.

Wang Yuncai, 2003. The study on recreation-scape planning and landscape ecological protection of urban-rural 
fringe: A typical case study of Beijing. Geographical Research, 22(3): 324-334. (in Chinese)

Wei Fang, 2017. Greener urbanization? Changing accessibility to parks in China. Landscape and Urban Planning, 157: 542-552.

Wei Fengqun, Xi Yueting, Shu Tian Cole, 2016. Development concept and strategy of urban recreation space in the perspective of spatial justice: Based on the experience of United States. Journal of Human Settlements in West China, 31(5): 51-56. (in Chinese)

Wong Koon Kwai, Yu Xiaojiang, 2012. Recreation conflict perception among visitors to Tuen Mun Park, Hong Kong, China: Outgroup evaluation, resource specificity, and lifestyle tolerance. Managing Leisure, 17(4): 349-362.

Wu Bihu, 2001. A study on Recreational Belt Around Metropolis (ReBAM): Shanghai case. Scientia Geographica Sinica, 21(4): 354-359. (in Chinese)

Wu Bihu, Dong Lina, Tang Ziying, 2003. A study on categories and attributes of public urban recreational spaces. Chinese Landscape Architecture, (5): 49-51. (in Chinese)

Wu Bihu, Jia Jia, 2002. Developing and managing urban waterfront tourism/recreational spaces: A case study of Wuhan City, China. Geography and Territorial Research, 18(2): 99-102. (in Chinese)

Wu Chengzhao, 1995. The history, theory and approach of urban recreation planning in W-Europ. Urban Planning Review, (4): 22-27, 33-63. (in Chinese)

Wu Zhijun, Tian Fengjun, 2012. The analysis of urban recreational space shape characteristic and influencing factors based on space syntax: Taking Nanchang as an example. Economic Geography, 32(6): 156-161. (in Chinese)

Wu Zhiqiang, Wu Chengzhao, 2005. Urban Recreation and Tourism Planning Studies. Beijing: China Building Industry Press. (in Chinese)

Xiao Guirong, Song Wenli, 2008. Structural optimization of urban recreational space in Dalian, China. China Population, Resources and Environment, 18(2): 86-92. (in Chinese)

Xiao Xing, Du Kun, 2011. A study on recreationists' satisfaction of Guangzhou city parks. Human Geography, 26(1): 129-133. (in Chinese)

Xiao Yang, Wang Zheng, Li Zhigang et al., 2017. An assessment of urban park access in Shanghai: Implications for the social equity in urban China. Landscape and Urban Planning, (157): 383-393.

Xin Changchang, Hu Minmin, 2016. Analysis of recreation space quality based on AHP: Taking Imperial Street of Southern Song Dynasty in Hangzhou City as an example. Journal of Hefei University of Technology (Social Sciences), 30(5): 120-125. (in Chinese)

Yan Shanshan, Liang Liuke, Yu Ruyi et al., 2016. The indicator system for the suitability evaluation of theme park construction in urban areas in China. Scientia Geographica Sinica, 36(2): 213-221. (in Chinese)

Yang Jianzhao, Zhu Jingjing, Ding Xinjun, 2018. Research on the development of urban recreational space system based on symbiosis theory. Ecological Economy, 34(3): 137-141. (in Chinese)

Yang Li, Ma Xianglian, 2015. The space structure feature of the recreational belt around metropolis of Changsha, China. Economic Geography, 35(10): 218-224. (in Chinese)

Yang Xiaojun, Fang Chuanshan, Hou Yezi, 2018. Optimization of ecological recreation area in Xi'an city based on ecological footprint. Geographical Research, 37(2): 281-291. (in Chinese)

Yang Yan, Shi Guang, 2016. Analysis and research on the accessibility of outdoor public recreation space in Qingdao, China. Agriculture Network Information, (3): 16-19. (in Chinese)

Ye Shengtao, Bao Jigang, 2009a. ROP-ENCS: A classification framework for the research of urban recreational spatial morphology. Tropical Geography, 29(3): 295-300. (in Chinese)

Ye Shengtao, Bao Jigang, 2009b. Description of urban recreational spatial morphology: Field model or feature model. Geography and Geo-Information Science, 25(3): 99-102. (in Chinese)

Ye Shengtao, Ye Tuo, Wu Xueming, 2015. Research for administrative department reform of urban recreation space. Journal of South China University of Technology (Social Science Edition), 17(3): 43-48, 107. (in Chinese)

Yi Jiandong, Horton Peter, 2015. The integration of three types of lands: A new approach to the provision of public sport and recreation areas in China. The International Journal of the History of Sport, 32(10): 1291-1307. 
Yin Zhenggang, Li Xueying, Bai Yang, 2010. A spatial analysis of the image of urban recreation sites: A case study on Zhengzhou. Areal Research and Development, 29(5): 79-84, 88. (in Chinese)

Yu Changming, Zhang Tianyao, 2015. Recreation space strategic planning of global cities and their experience for Beijing, China. Planners, 31(8): 5-11. (in Chinese)

Yu Kongjian, Duan Tiewu, Li Dihua et al., 1999. Landscape accessibility as a measurement of the function of urban green system. Planning Studies, 23(8): 7-10, 42, 63. (in Chinese)

Yu Sheng, 2003. Urban Tourism and Urban Recreation. Shanghai: East China Normal University Press. (in Chinese)

Yu Sheng, He Shanbo, 2003. The research of urban RBD distribution. Human Geography, 18(4): 10-15. (in Chinese)

Yue Bangjia, Lin Aiwen, Sun Cheng, 2016. Analysis on accessibility of urban park green space based on public transport system: The case study of park green space in Wuhan's urban center. Geomatics and Spatial Information Technology, 39(12): 60-63, 67. (in Chinese)

Zhang Haixia, 2010. Social policy and provision for public recreation: Towards the spatial body for governmental action. Tourism Tribune, 25(9): 20-26. (in Chinese)

Zhang Hua, Chen Bo, Sun Zhi et al., 2013. Landscape perception and recreation needs in urban green space in Fuyang, Hangzhou, China. Urban Forestry \& Urban Greening, 12(1): 44-52.

Zhang Huanzhou, Shen Xuwei, Gao Jing, 2011. Spatial structure of the leisure zone in urban waterfront: A case study of the Grand Canal in downtown Hangzhou, China. Geographical Research, 30(10): 1891-1900. (in Chinese)

Zhang Yishan, Xia Jian, 2008. The protection and reuse of urban industrial heritage by integrating into public recreation spaces system. Industrial Construction, 38(4): 27-30, 49. (in Chinese)

Zhu He, Liu Jiaming, Chen Chen et al., 2015. A spatial-temporal analysis of urban recreational business districts: A case study in Beijing, China. Journal of Geographical Sciences, 25(12): 1521-1536.

Zhu He, Liu Jiaming, Li Le et al., 2014. Research progress on urban recreational business district (RBD) in China. Progress in Geography, 33(11): 1474-1485. (in Chinese)

Zhu He, Liu Jiaming, Liu Huaxian et al., 2017. Recreational business district boundary identifying and spatial structure influence in historic area development: A case study of Qianmen area, China. Habitat International, 63: 11-20. 